\title{
PLANNING AND PROJECTS: THREE VISIONAIRES FRIEDMANN, J., TRUEBA, I. AND RAMOS, A.
}

De Nicolas de Nicolas, Victor Luis; Cazorla Montero, Adolfo

Universidad Politécnica de Madrid

It is impossible to talk about planning as a scientific meta-discipline without mentioning one of the most influential worldwide figures in the second half of the twentieth century: John Friedmann. His contribution to the planning concept on his "Planning as Social Learning" theory is still very relevant.

This paper shows the intellectual connection between Friedmann and Angel Ramos and Ignacio Trueba, two of the Spanish intellectual drivers in the engineering project knowledge area, who contributed to founding the Engineering Projects Spanish Association.

The three of them share a broad vision of the project and abandon the "blue print" planning model. They also see the project as a transformational tool that requires a different planning style to the one which prevailed in the 70 s - both in public and private domains. They were pioneers in structuring Knowledge / Action in a different way, both in academic institutions where disciples helped to bring about change- and with direct action via projects.

Keywords: Projects; Planning, Social Learning; Knowledge / Action; Three visionaires

\section{PLANIFICACIÓN Y PROYECTOS: TRES MAESTROS FRIEDMANN, J., TRUEBA, I. Y RAMOS, A.}

Resulta imposible hablar de Planificación como metadisciplina científica sin citar una de las figuras de mayor relevancia e influencia mundial en la segunda mitad del siglo XX: John Friedmann. Su aportación al pensamiento de la Planificación en su "Planning as Social learning" continua siendo muy relevante.

Esta comunicación muestra la conexión intelectual entre Friedmann y dos de los impulsores intelectuales del Área de conocimiento de Proyectos de Ingeniería y fundadores, con otros, de la Asociación Española de Ingeniería de Proyectos: Ángel Ramos e Ignacio Trueba.

Los tres coinciden en su visión amplia del Proyecto y en la superación, por lo tanto, del modelo "blue print". Al mismo tiempo ven el Proyecto como elemento transformador que precisa un estilo de Planificación diferente del que en esos momentos -década de los 70 del siglo pasado- imperaba, tanto en las administraciones públicas como en las empresas. Ellos fueron pioneros de otros modos de vertebrar el Conocimiento/Acción tanto en la Academia, preparando una pléyade de discípulos que contribuyeron al cambio, como en la Acción directa a través de Proyectos.

Palabras clave: Proyectos; Planificación; Social Learning; Conocimiento/Acción; Tres maestros 


\section{Introducción}

Los conceptos de Planificación y Proyectos han estado y están muy unidos tanto en el ámbito público como en el privado en lo que podría llamarse "operatividad". También en la Academia con sus líneas de investigación y de docencia aparecen conceptualmente entrelazados. Es un clásico en las universidades tecnológicas la conexión entre Plan, Programa y Proyecto de forma que el Proyecto es el instrumento operativo de máxima concreción de los Planes y de los Programas (Fig. 1).

Figura. 1: Trueba et al, 1995

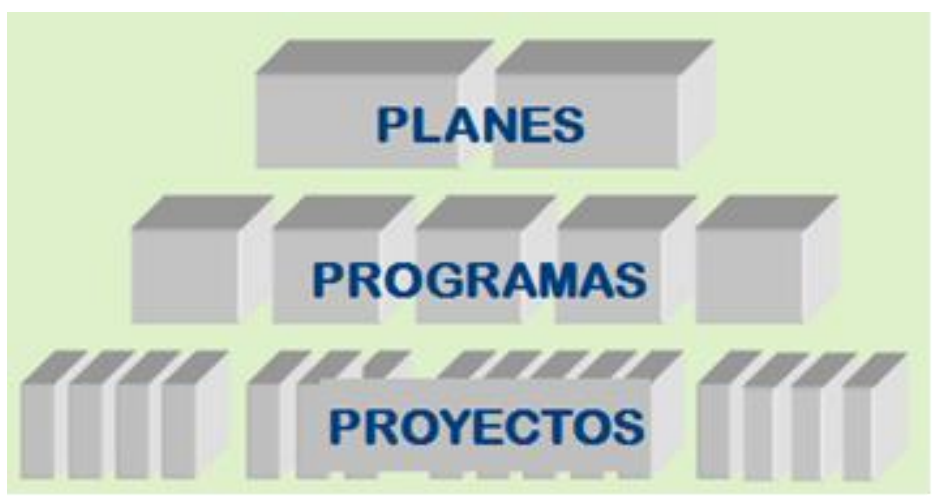

Parafraseando a Manjón con unas formas de decir que reflejan el sabor de lo añejo, en lo que tiene de perenne: "no es mejor maestro el que más sabe, ni siguiera el que más instruye, sino el que mejor educa, esto es, el que tiene el raro don de saber hacer hombres dueños de sí y de sus facultades, el que asocia su trabajo al de los alumnos y les hace participar de las delicias de la paternidad de sus conocimientos" (De Arce, 2002).

Las figuras de John Friedmann, Ángel Ramos e Ignacio Trueba, pertenecen a este grupo relevante de personas innovadoras en el ámbito de la Planificación y Proyectos que desde sus Departamentos universitarios supieron además crear un estilo de pensar y de hacer. En palabras de Ibáñez Martín "estilo, procede sin apenas variación, del latín y del griego, en los que significaba el punzón, el estilete que se usaba para marcar las letras en las tablas recubiertas de cera, que eran el antecedente del papel. $Y$ al igual que toda máquina de escribir - dicen las novelas policíacas- tiene sus señas de identidad, también las tenían tales punzones, por la que se podría descubrir al autor del escrito. De ahí que hoy estilo no se refiera ya al instrumento material sino a la idiosincrasia, a la personalidad, al modo específico de ver las cosas o de comportarse de una persona o de un tipo de personas." (Ibáñez Martín, 1995). Desde el inicio del Department of Urban Planning de la Universidad de California, Los Ángeles, por el doctor Friedmann en 1969, supo crear todo un Pensamiento en Planificación del que nos hemos beneficiado universitarios de los cinco continentes; Ángel Ramos e Ignacio Trueba fueron las dos cabezas señeras que iniciaron el Departamento de Proyectos y Planificación Rural en la Universidad Politécnica de Madrid a partir de 1985 y contribuyeron, desde el primer momento, a la creación de AEIPRO (Asociación Española de Ingeniería de Proyectos) alrededor del Área de Conocimiento de Proyectos de Ingeniería e impulsaron unas reuniones periódicas que, con el tiempo, se consolidarían en los Congresos Internacionales de Ingeniería de Proyectos cuya XVIII edición se celebra este año 2014.

Julián Marías decía, a propósito de lo anterior, que estaba convencido que la filiación intelectual funciona de modo inverso a la biológica personal. Esta, decía, la proclaman y reconocen los padres, al afirmar su paternidad sobre sus hijos, mientras que en el caso de la intelectual ha de ser el hijo quien reconozca públicamente al padre y admita la deuda con el 
maestro, y muestra las influencias que ha recibido y le han llevado a ser quien es y como es (Carpintero, 2008).

A continuación se desarrolla lo que, a juicio fundamentado de los autores, supone el hilo conductor del pensamiento de los tres y que se resumen en cuatro conceptos: 1) El proyecto moderno, 2) La reivindicación ecológica y 3) Conocimiento/Acción en la postmodernidad.

\section{Conceptos claves de partida en los tres autores}

La idea de que el conocimiento científico sobre la sociedad podía aplicarse a su perfeccionamiento, apareció fundamentado, por primera vez, durante el siglo XVIII. En este periodo dominaba el pensamiento de que cualquier idea valiosa tenía que ser práctica, midiendo con rigor matemático cuáles eran sus consecuencias (Friedmann, 1987). Las ideologías utópicas modernas nos presentaban la ciencia, la técnica y la planificación como instrumentos infalibles para el control racional de la naturaleza y de la sociedad (Llano, 1988). Estas ideologías, aunque con diferencias según los distintos países, tienen una raíz común de carácter filosófico que ha venido a llamarse modernidad (Spaemann, 2004).

\subsection{El proyecto moderno y su repercusión en Ingeniería: Modelo Blue Print}

En la Modernidad, la planificación se veía como algo inseparable del poder (Shumacher, 1976). Asociado a este concepto de planificación se encuentra el llamado proyecto moderno -proyecto blue print- que se fundamenta en la ingeniería, en la racionalidad científica con enfoques descendentes: blue print is top-down-approach como concepto vinculado a una racionalidad objetiva y reduccionista que hunde sus raíces en los campos de la ingeniería y la construcción (Bond \& Hulme, 1999). Desde este enfoque se diseñaron los primeros modelos de planificación del desarrollo (Mannheim, 1949) (Lindbloom, 1977) (Etzioni, 1968).

La influencia de Saint Simon (1760-1825) fue manifiesta en la visión de este proyecto moderno aportando enfoques complementarios desde la Planificación científica. Saint Simon sugería la consideración de una imagen de la sociedad, en la que los "científicos e ingenieros" como los mejores conocedores de las leyes orgánicas de la sociedad, trazarían el futuro de acuerdo con un plan global (Friedmann, 1987).

Esta visión inspirada por las ciencias de la ingeniería y por el idealismo de los ingenieros Saint-Simonianos (Wolf, 1981), implicó importantes consecuencias en la visión del hombre en las relaciones con la naturaleza, influyendo en los primeros modelos de desarrollo y en los proyectos de ingeniería clásicos (Hayek, 1955).

Ese diseño planificado de la sociedad recibió el nombre de planificación social dentro del proyecto moderno, de forma que "cada hombre es un individuo más dentro de un organismo social al que ha de subordinarse, o ser subordinado, que debe darse por satisfecho... con las abundantes concesiones de que goza dentro del sistema vigente, acreditado con firmeza como el mejor de los posibles" (Ramos, 1993).

\subsection{La reivindicación ecológica: En busca del equilibrio perdido}

La peculiar dignidad que el pensamiento moderno atribuye al hombre procede inmediatamente de la idea de dominio. Una de las consecuencias que este enfoque ocasionó es que "el proyecto moderno no contempla el cuidado y el respeto, que son cuestiones menores a la luz de la eficacia y de la utilidad, normas supremas con las que no debe interferir lo vital: la solidaridad con la naturaleza no existe, puesto que ésta ha de ser dominada" (Ramos, 1993).

La reivindicación ecológica plantea por lo tanto, con sus luces y sombras si el progreso técnico, indudablemente conseguido, está de acuerdo con el progreso ético del hombre. 
En la figura 2, se sintetiza el esquema de una sesión de trabajo en Cervera de Pisuerga (1984) en donde con una visión comprensiva de lo que habían sido las relaciones del hombre con la naturaleza se mostraba como, Don Àngel, veía en el futuro un conocimiento un saber- como compresión de la naturaleza que iría más allá de lo que había ocurrido desde el siglo XVII (Cazorla, 1999).

Fig. 2.- (Ramos, 1984)

SABER ES SABER SABER ES PODER SABER ES HACER SABER ES TENER SABER ES ADMINISTRAR

(hasta el siglo XVII) (siglos XVII-XIX) (Siglos XIX-XX) (segunda mitad del siglo $X X$ ) (final del siglo $\mathrm{XX}$ )

\section{El futuro está en volver al "Saber es Saber" con matizaciones. Unos siglos "contemplación", unos pocos siglos de "explotación" con la inflexión de "administración" (con una variable económica)}

\section{El matiz consiste en que "SABER ES COMPRENDER"}

Se trata en definitiva de buscar una salida a lo que señalaba Schumacher: "podemos decir que el mundo moderno ha sido modelado por la tecnología. Se tambalea de crisis en crisis" (Schumacher, 1978).

Entre los signos visibles de destrucción apunta Schumacher el deterioro del solar donde se desarrollan las actividades de los hombres. Por eso tiene mucho sentido la visión de Ramos (Cazorla, 1999) cuando apunta al "quiebro histórico en la idea de progreso con la que ha transcurrido la segunda mitad del siglo $\mathrm{XX}$, un punto de inflexión a partir del cual comenzará un proyecto que reivindica el respeto hacia la naturaleza como parte de la preocupación real por las condiciones de vida de las personas que hoy habitan este planeta y de las que lo habitarán en el futuro".

Esta reflexión permite situar dos elementos clave del proceso conceptual que se está describiendo: en primer lugar, un Proyecto que reivindica el respeto hacia la naturaleza y en segundo lugar, las condiciones de vida de las personas actuales y futuras. La conexión de estos dos elementos tiene su respuesta en Trueba (2002 y 1995) cuando, refiriéndose a Ángel Ramos comenta: "Su conocimiento sobre los recursos naturales, su inquietud científica e investigadora y sobretodo su colosal sensibilidad y respeto por la naturaleza han contribuido a mi formación (Trueba, 2002). Como consultor de FAO durante años compaginando dicho trabajo con su posición de catedrático de proyectos en la Universidad Politécnica de Madrid, tuvo ocasión de participar activamente en el diseño de nuevas metodologías de proyectos que FAO pondría en marcha en la década de los 70 (FAO, 1971) y que luego adaptaría a entornos educativos y empresariales con el ánimo de impulsar una concepción del proyecto reivindicador del respeto hacia la naturaleza e integrador en la toma de decisiones de futuros empresarios (Trueba, 1995).

\subsection{Conocimiento/Acción en la postmodernidad: Elementos de un nuevo proyecto}

$\mathrm{Si}$ el proyecto moderno nos enmarcaba lo antiguo, aunque con resistencias, de ser abandonado, y la reivindicación ecológica planteaba la crisis del sistema, en este apartado se pretende iniciar la construcción intelectual del nuevo proyecto en una 
postmodernidad emergente con los elementos clave que, a juicio de nuestros tres maestros, deben contener.

El término postmodernidad aparece, por primera vez, en la obra de Toynbee "A Study of History" (Toynbee, 1987) haciendo referencia a un cambio de paradigma respecto a la modernidad. Surge como reacción, ya señalada anteriormente, por el fracaso de la modernidad tecnocrática, la idea del progreso indefinido y el varapalo ocasionado por la reivindicación ecológica que postula la búsqueda de un equilibrio perdido (Cazorla, 2013).

Desde principios de los años 90 diferentes autores hacen referencia al surgimiento de esta postmodernidad como un efecto cultural e ideológico, con nuevos valores y tendencias que reclama nuevos modos de pensar y de actuar en contraste creciente con los del pasado (Ballesteros, 1989). En esta nueva etapa se refleja la falta de novedad de la sociedad capitalista industrial, que se convierte en una sociedad "vieja". Algunos autores denominan este cambio como postmodern sensibility to difference (Cloke, Philo \& Sadler, 1991) (Philo, 1992).

Como el concepto tradicional de Planificación ha estado profundamente relacionado con el llamado proyecto de corte moderno, también llamado euclidiano, una de las tentaciones intelectuales que surgieron se sintetizaron en un "euclidiano o nada" (Cazorla, 2006). Una de las maneras de resolver este dilema fue la definición de Friedmann (1987) del concepto de Planificación como aquella práctica profesional que busca específicamente conectar las formas de conocimiento con las formas de acción que este autor plantea en un relevante y premiado artículo como el mejor del año en USA de este autor (Friedmann, 1993) lleva a reconcebir la planificación como algo distinto de la ingeniería donde los medios están eficazmente relacionados con los objetivos y los proyectos trazan el curso de la acción.

¿Dónde encontrar el equilibrio entre la necesidad de desarrollar proyectos partiendo de una concepción no euclidiana de planificación? ¿Qué conocimiento/ acción serían válidos en este contexto postmoderno? Para enfocar adecuadamente la cuestión, remontarse a Aristóteles y la interpretación que Llano (1988) hace, parece pertinente.

No siempre se ha actuado bien y ahí está el modelo de desarrollo generado; se hace necesario matizar que la valoración de las acciones y su consideración de buenas o malas, no es algo posterior o resultante de las acciones mismas, sino constitutivo de ellas. El fin de hacer y del actuar es de distinta índole. El primero consiste en un producto; mientras que el fin del actuar no puede ser distinto del que actúa. Con Aristóteles concluimos que la buena praxis misma es fin. La praxis -la acción humana- no nos remite como la producción a la perfección de una obra externa, sino a la perfección lograda por el agente (Llano, 1988).

La recuperación de este verdadero concepto de acción en este nuevo periodo histórico tiene un primer componente, de ese nuevo estilo de planificación, que afecta tanto a las relaciones del hombre con la naturaleza como con los otros hombres y nos lleva a decir, con Spaemann, que "lo injusto por naturaleza no puede ser al mismo tiempo lo óptimo funcional" (Spaemann, 1983). En otras palabras, no debemos hacer necesariamente todo lo que técnicamente podemos hacer (Ramos, 1993).

Dentro de la acción, la noción de cuidado es incluida por Llano como otra de las claves de esta nueva sensibilidad postmoderna cuando dice "la racionalista y unidimensional actitud de dominio ha de ser sustituida por esa originaria unidad de contemplación y acción que es la epimeleía, el cuidado" (Llano, 1988).

En coherencia intelectual con este planteamiento que Friedmann (1993) describía en el artículo citado y con Alejandro Llano escribe Ramos (1993) sobre el cuidado como concepto guía para la acción: "El cuidado nunca procede con prepotencia ni pretende irrumpir agresivamente en la realidad". 
Al concepto de cuidado, en el plano operativo, le corresponde el término respeto en el plano del conocimiento. Siguiendo a Millán Puelles: "El respeto a la naturaleza son también necesidades humanas naturales exigencias primarias de nuestro modo de ser y cuya satisfacción se nos presenta como un cierto valor en si y por si (Millán, 1984).

Figura 3: Autores a partir de los tres maestros

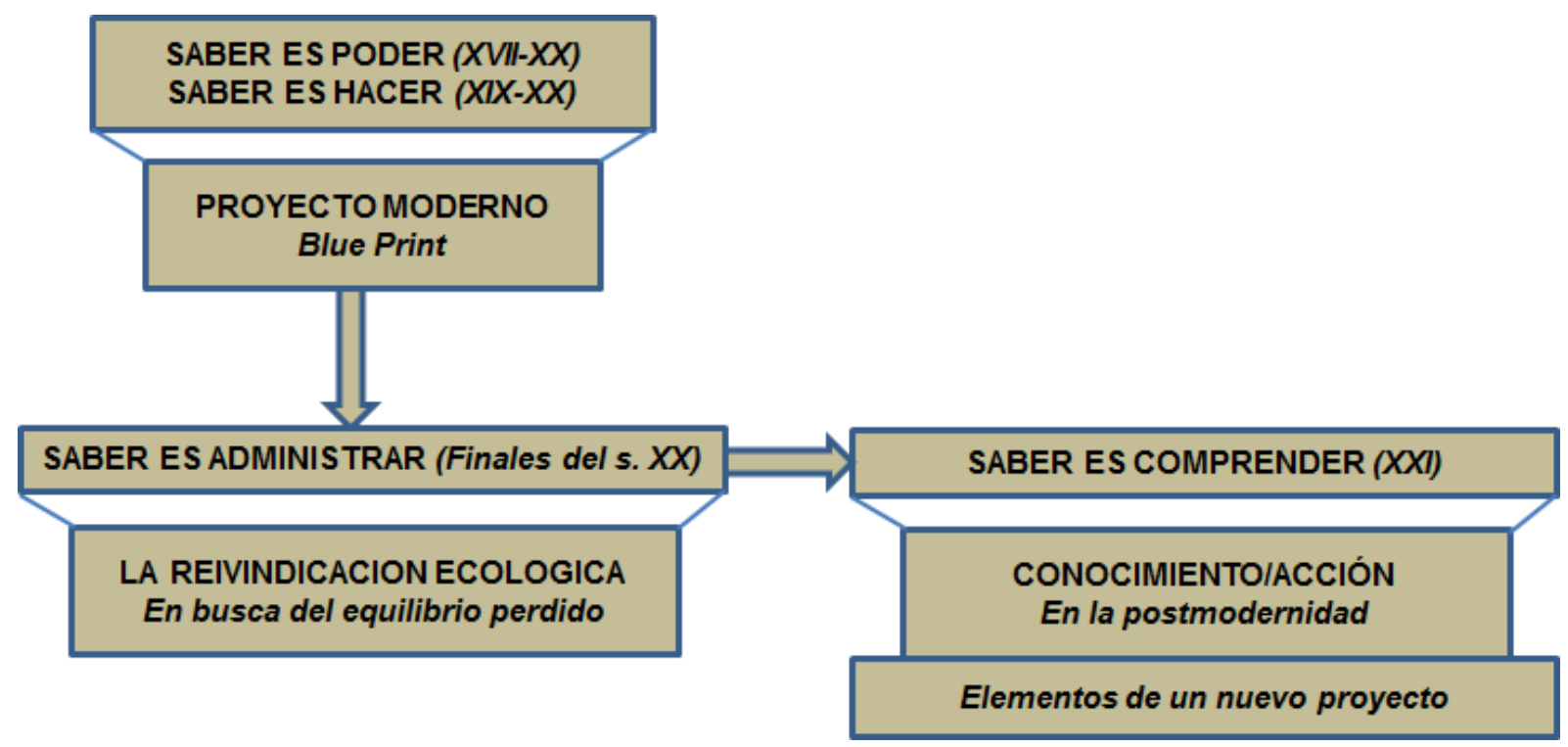

En síntesis podemos avanzar y en la figura 3 se refleja que, la restauración de estas coordenadas de forma, nos llevará en el plano operativo a una visión integrada en la formulación de proyectos (Trueba, 1995) consecuencia de un hacer bien, pero con un planteamiento de fondo de respeto, fruto del conocimiento y de cuidado intrínsecamente unido a la acción. Llevar a un modelo de planificación postmoderno esta new postmodern sensibility requiere coherencia -valores- que permitirán descubrir y redimensionar espacios vitales desde la participación y el compromiso, entendido éste como "la aportación personal" de cada uno de los agentes que están dentro o asociados a un proyecto (IPMA, 2010).

\section{Friedmann, Ramos, Trueba: Sus aportaciones intelectuales específicas}

Si en el apartado anterior se ha fundamentado la base intelectual de los tres autores, a continuación se desarrollarán sus aportaciones específicas en el ámbito de la Planificación y los Proyectos.

\subsection{John Friedmann: Planning as Social learning}

El concepto de Aprendizaje Social aparece en la literatura internacional como un modo de desarrollar un concepto de Planificación alternativo a los modelos de Planificación existentes en el ámbito público. El libro que Friedmann propone a la sociedad se llamaría "Retracking America: A theory of transactive Planning", y se publicó en 1973; ocasionó una verdadera conmoción intelectual en los ambientes académicos norteamericanos ofreciendo un nuevo sistema que vendría a reemplazar el modelo en quiebra que la sociedad americana post industrial estaba viviendo. Su crítica a la planificación asignativa, vigente en ese momento, que se ocupaba de la distribución central de una serie de recursos escasos entre las diferentes personas, pasaría a ser sustituido por una planificación innovadora de gestión, con un elemento clave, la relación personal que debe existir entre experto y cliente (Cazorla, 2001). 
El proceso epistemológico desarrollado por Friedmann, a partir de esos años, continúa explorándose en sus amplias ramificaciones. En contra de la visión de Karl Popper, que considera la ciencia como una cuestión de hipótesis refutadas que si resisten las pruebas se convierten en más robustas, Friedmann propone que en la práctica social debe ocurrir justo lo contrario. El papel del planificador no es, en ningún caso, la construcción de teorías sino la innovación social de forma que, se situaría así como el profesional responsable de conseguir movilizar los recursos y buscar concentrar las energías públicas y privadas en soluciones innovadoras para los desafiantes problemas del dominio público (Friedmann, 1993).

Los procesos de una Planificación como Aprendizaje Social funcionan con el supuesto central de que todo aprendizaje efectivo proviene de la experiencia de cambio de la realidad. La población afectada por un proyecto participa activamente en la Planificación, mediante sus propios comportamientos, actitudes y valores que generan acciones encaminadas a integrar el conocimiento experimentado (Hulme, 1989) de la población afectada, junto con el conocimiento experto del Planificador proporcionando un aprendizaje mutuo (Argyris \& Shön, 1978).

Este respeto por las personas implicadas -trabajar con ellas- ha permitido desarrollar en el tiempo procesos metodológicos de participación, negociación y planteamientos éticos profundos con éxitos profesionales y académicos notables (De los Ríos, 2013).

\section{2. Ángel Ramos: Planificación física y Proyectos.}

John Friedmann se convirtió en director del Programa de Planificación Urbana de la Universidad de California, Los Ángeles, en 1969; fue a partir de ese momento en que su experiencia práctica de consultor de gobiernos e instituciones en varios países cristalizó con unas enseñanzas que permitirían beneficiarse a decenas de alumnos de los cinco continentes. Existe un recorrido similar en la figura de Ángel Ramos que durante la década de los sesenta desarrolló una intensa actividad profesional, relacionada con la restauración ambiental en proyectos de obra civil y al conseguir en 1971 la cátedra de Planificación Física y Proyectos de la Escuela de Montes en la Universidad Politécnica de Madrid vuelca todo su dinamismo en la preparación de profesionales y profesores buscando el equilibrio entre la tecnología agresiva y la conservación a todo trance, propugnada por algunos grupos ecologistas (Fernandez-Galiano \& González Alonso, 1999).

Sus reflexiones de esos años cristalizaron en una publicación muy trascendente: Planificación física y Ecología (1979) que continúa como referencia imprescindible para los trabajos de ordenación del medio natural.

Àngel Ramos es junto con Ramón Margalef y Fernando González Bermúdez, tres pioneros de la introducción de un nuevo modo de pensar, hacer y desarrollar acciones humanas proyectos- en la naturaleza, "La traducción práctica de este nuevo planteamiento tropieza con importantes dificultades... Entre las primeras está la ineludible obligación de satisfacer, en tantos campos, las necesidades del hombre y de seguir, por lo tanto, desarrollando proyectos. Las posturas obstruccionistas parecen desconocer esta realidad, al igual que un puro crecimiento económico ignora los efectos laterales de un proyecto" (Ramos, 1979).

Podría afirmarse que lo mismo que Friedmann postula una metodología de trabajo con la gente mediante el cruce del conocimiento experto y experimentado, Ramos apuesta por un diálogo con la naturaleza "saber ver, saber apreciar, saber valorar" con vistas a un desarrollo sostenible que hunde sus raíces en la consideración de las variables del medio biótico y abiótico que permitan encontrar "el difícil punto justo que tantas veces, por otra parte, no estará en uno de los extremos pero tampoco será el punto medio. Frente a los reduccionismos de uno y otro signo, aporta la visión integrada de los problemas y de las soluciones, frente a urgencias y trepidaciones, la proyección en el tiempo, los plazos largos" 
(Ramos, 1993). Por eso la naturaleza ha de ser respetada, tiene su propia fisonomía y un destino anterior: de ahí la importancia de descubrir, en ese diálogo silencioso, los ritmos naturales $\mathrm{y}$, al mismo tiempo, desarrollar proyectos que integran esa valiosísima información.

\subsection{Ignacio Trueba: Proyectos y Planificación Rural.}

Cuando consiguió la cátedra de Proyectos de Ingeniería en la Universidad Politécnica de Madrid en los primeros años de la década de los 70, Ignacio Trueba había desarrollado ya una ingente tarea profesional tanto en el Ámbito Público, en la década anterior con la Formulación de los llamados Planes Provinciales del Ministerio del Interior del Gobierno de España, como de Consultor de FAO y Naciones Unidas, labor que continuaría desde el Departamento de Proyectos y Planificación Rural que se creó a mediados de la década de los 80 como consecuencia de la nueva organización departamental de la Universidad española (Trueba, 2002).

Esa experiencia profesional resultó muy enriquecedora para adelantar metodologías de Formulación y Evaluación de proyectos partiendo de una concepción amplia del concepto de proyecto que,"así entendido involucra muchas más disciplinas que las puramente tecnológicas, entrando de lleno en el área del desarrollo económico, la política social y la regeneración ambiental" (Trueba \& Morales, 2011). Esta "forma de ver" los proyectos en su amplio sentido lleva, en el desarrollo conceptual del Doctor Trueba, a una forma sistemática de toma de decisiones conocido como el Ciclo de los proyectos. "Valga la expresión, al igual que los seres vivos, el proyecto, nace, crece, se desarrolla, fructifica y muere. Tiene un ciclo vital" (Trueba, 1985, 1982).

El concepto de Planificación Rural conectado con los proyectos supuso una apuesta intelectual innovadora adelantándose años a lo que la Comisión Europea llamaría Iniciativa Comunitaria LEADER (Liaisons Entre Activités de Développement de l'Économie Rural), como impulso de un desarrollo rural a través de proyectos, con unas metodologías de participación social y de respeto al medio ambiente que al día de hoy siguen funcionando.

El papel de las comunidades rurales como protagonistas de su propio desarrollo a través de metodologías bottom/up de "abajo a arriba", han permitido durante décadas llevar a cabo proyectos de ingeniería que vertebran y desarrollan territorios rurales uniendo a su tradicional componente técnico-económico las otras características ya mencionadas (De los Ríos, 2013).

\section{Lo común de los tres: Los valores del profesional de la planificación y proyectos}

Para enmarcar este apartado parece adecuado recordar algo esencial de carácter metafísico. Desde Aristóteles se coincide intelectualmente en que los modos fundamentales de ser, a los que se reduce toda realidad, son la sustancia y los nueve tipos de accidentes llamados también predicamentos o categorías. Como el ser se refleja en el conocimiento y en el lenguaje, a esos modos de ser corresponden los diversos tipos o géneros de predicados que pueden atribuirse, de ahí el nombre de predicamentos (Alvira et al, 2001).

Todos los accidentes tienen en común inherir en la sustancia, el ser en un sujeto, pero además cada accidente posee una esencia propia y, por eso, determina a la sustancia de un modo original (Alvira et al, 2001). De los nueve géneros de accidentes hay unos que se denominan extrínsecos porque no afectan la sustancia, en y por si misma, sólo de manera externa; por ejemplo, la situación (situs), el donde (ubi), la posesión (habitus), el cuándo (quando). Hay otros accidentes que si afectan intrínsecamente a la materia: entre estos, queremos centrar nuestra investigación en el accidente relación. 


\subsection{La relación, categoría determinante de la sustancia para la acción}

La relación es el accidente cuya naturaleza consiste en la referencia u ordenación de un ente hacia otro distinto de él. La clave de este accidente es, como un salir de sí hacia otro. La realidad propia de la relación es, por tanto, imperfecta y debilísima al consistir en un puro "respecto a" (Alvira et al, 2001).

Hasta aquí nuestra reflexión metafísica para, volviendo a nuestros tres maestros, mostrar, como en ellos incide esta base metafísica ya que, a pesar de poseer una realidad muy débil, el alcance de este accidente es muy considerable y en los autores que estamos estudiando constituye una de las claves que podemos descubrir comunes a los tres y próximas también en sus desarrollos.

Las relaciones del hombre/naturaleza (Ramos), la relación entre conocimiento experto/experimentado (Friedmann) y las relaciones urbano/rurales (Trueba) son seis conceptos perfectamente vertebrados por estos maestros, que cumplen la máxima clásica de que el saber es en el fondo saber relacionar. En páginas anteriores se han desarrollado estos conceptos propios que tienen una base común. El hombre y su acción con sus semejantes (Friedmann), con la naturaleza (Ramos) y con contextos espaciales (Trueba). Sobre esta base lo que pretenden los tres es cambiar la realidad según un profundo sentido de cómo es esa realidad.

\subsection{Los profesionales de la planificación y los proyectos: Algunos principios inspiradores}

Pensamos que ha quedado claro a lo largo de estas páginas un elemento nítido de nuestros tres maestros: un desempeño profesional durante años en ambientes muy variados. En el caso de J. Friedmann como consultor internacional; A. Ramos en cambio, trabajó en una empresa restauradora de paisajes e I. Trueba alternó trabajos de responsabilidad como gestor público en planes y programas con consultorías internacionales en FAO y Banco Mundial. Después de años de trabajo intensos consiguieron incorporarse a la universidad en posiciones relevantes que les llevaron a aportaciones intelectuales sobresalientes y muy vinculadas a esa realidad vivida en contextos culturales muy dinámicos. Nada mejor que perfilar aquí algunos principios guía inspiradores para los profesionales de la planificación y los proyectos en el contexto postmoderno en que nos encontramos. Llano en su libro sobre la Nueva Sensibilidad (1988) aporta una síntesis que apreciamos compartida por estos tres maestros.

A) Principio de solidaridad. Si el proyecto moderno ha creado espacios sociales incompatibles, una planificación y un proyecto postmoderno reclama ámbitos sociales compatibles, primando espacios de solidaridad que tienen su fundamento más sólido en la dignidad de la persona y que tienen una proyección en "el cuidado y el respeto de la naturaleza, seres vivos, procesos naturales, paisajes se prolongan hasta la condición del hombre y de los hombres entre sí (Ramos, 1993).

Trueba (2002), concreta ese pensamiento cuando escribe: "hace falta una solidaridad global que diariamente nos recuerde los compromisos y promueva una acción de desarrollo humano basado en la libertad".

B) Principio de integralidad. Aunque, el reto de la interdisciplinariedad conceptualmente parece asumido, queda un campo pendiente por desarrollar incoado en el pensamiento de Ramos, Trueba y Friedmann. Este último acuñó un concepto muy relevante al expresar que "los empresarios de la planificación son principalmente movilizadores de recursos que buscan concertar energías públicas y privadas en soluciones innovadoras para los desafiantes problemas de dominio público. Pero tal planificación está orientada a valores 
especiales y no al beneficio. Es explícitamente normativa en su intención" (Friedmann, 1992).

Aquí aparece con toda su fuerza la integralidad en su dimensión "integral" desde los valores -desde la ética más que desde la técnica- de forma que, esos planificadores aunque sean libres de elegir, la acción en el dominio público deberá justificarse como la que favorece la causa del florecimiento humano y la diversidad -también ecológica- en todo el mundo (Friedmann, 1993).

C) Principio de complementariedad. Concluimos con Llano (1988), que frente a la dominante estrategia de conflicto, que confunde lo que es distinto con lo que es contrario, asciende otro modo de pensar que no es excluyente sino que afirma la composibilidad de las diferencias. Que mejor complementariedad que la espacial, en donde el mundo rural se presenta como equilibrador de un mundo urbano que ha generado más consecuencias nocivas, para el medio ambiente, en general y, para el hombre en particular.

\section{Conclusión}

Nuestros tres maestros supieron, quizá, sin formularlo expresamente, desarrollar su pensamiento en principios vertebradores como los enumerados pero, lo más importante, a modo de conclusión -y conectando con el principio de esta comunicación- lo vivieron y lo enseñaron a generaciones futuras que ahora, en la actualidad, lo desarrollan de forma natural como un tesoro recibido casi sin darse cuenta, como por "ósmosis", según reflexión de Santiago González Alonso (Cazorla, 1999) refiriéndose a D. Ángel Ramos.

\section{Referencias}

Alvira, R., Clavel, L. Melendo, T. (2001). Metafísica. Pamplona: EUNSA.

Azgyris \& Shön (1978). Organisational learning: a theory of action respective. Boston: MA Addison Wesley

Ballesteros, J. (1989). Postmodernidad: decadencia o resistencia. Madrid: Tecnos.

Bond, R. \& Hulme, D. (1999). Process Approaches to Development: Theory and Sri Lankan Practice. World Development 27 (8), 1339-1358.

Carpintero, H. (2008). Julian Marías: una vida en la verdad. Madrid: Biblioteca

Cazorla, A. (1999). La enseñanza de la planificación por un maestro: D. Angel Ramos. Libro homenaje a D. Angel Ramos Fernández (1926-1998). Madrid: Real Academia de Ciencias Exactas, Físicas y Naturales.

Cazorla, A. (2001). Presentación. Planificación en el ámbito público: del conocimiento a la acción. Madrid: INAP

Cazorla, A. (2006). Planificación para la sostenibilidad: proyectos de Ingeniería para un ámbito rural local. Madrid: ETSI Agrónomos.

Cazorla, A., de los Ríos, I., \& Salvo, M. (2013). Working With People (WWP) in Rural Development Projects: a Proposal from Social Learning. Cuadernos de Desarrollo Rural, 10 (70), 131-158.

Cloke, P., Philo, C., Sadler, D. (1991). Approaching human geography. London: Paul Capman.

De Arce, U. (2002). Manjón, educador. Pulso, 25, 87-101

Etzioni, A. (1968). The active society: a theory of societal and political processes. New York: New York Free Press.

FAO (1991). Formulación de proyectos de producción agrícola. Roma

Fernández-Galinano, E. \& González Alonso, S. (1999). Retrato de un hombre singular, en homenaje a Don Angel Ramos Fernández (1926-1998). Madrid: Real Academia de Ciencias Exactas, Físicas y Naturales, Academia de Ingeniería. 
Friedmann, J. (1973). Retracking America: A Theory of Societal Planning. City, NY: Anchor Press

Friedmann, J. (1987). Planning in the public domain: from knowledge to action. Princeton: Princeton University Press

Friedmann, J. (1993). Toward a Non-Euclidian Mode of Planning. Journal of American Planning Association, 482, Chicago

Hayek, F. (1955). The counter-revolution of science: studies on the abuse of reason. New York: New York Free Press

Hulme, A. (1989). Hulme, D. (1989). Learning and not learning from experience in rural project planning. Public Administration and Development, 9(1), 1-16.

Ibáñez Martín, J.A. (1995). Formación y estilo universitario. Revista española de pedagogía, 43(169), 335-344..

IPMA, (2010). NCB- Bases para la competencia en dirección de proyectos.v.3.1. Valencia: AEIPRO

Lindblom, C.E. (1977). Politics and markets: the world's political economic systems. New York: Basic Books

Llano, A. (1988). La nueva sensibilidad. Madrid: Espasa-Universidad.

Mannheim, K. (1949). Man and society: in an age of reconstruction: New York: Harcourt, Brace and Co., 1940

Millán, A. (1984). Léxico filosófico. Madrid: Rialp.

Philo, C. (1992). Neglected rural geographies: a review. Journal of rural studies, 8(2), 193207.

Ramos, A. (1979). Planificación física y ecología. Madrid: EMESA

Ramos, A. (1993) ¿Por qué la conservación de la naturaleza? Discurso en el acto de recepción en la Real Academia de Ciencias Exactas, Físicas y Naturales. Madrid.

Schumacher, E. F. (1993). Small is beautiful: A study of economics as if people mattered. Random House.

Spaemann, R. (1983). Ende der Modernität?. Philosphische essays. Sttugart.

Spaemann, R. (2004). Ensayos filosóficos. Madrid: Biblioteca Filosófica Ediciones cristiandad.

Toynbee, A. J. (1987). A study of history (Vol. 1). Oxford University Press.

Trueba, I. (1982). Teoría de proyectos: Morfología. Madrid: ETSI Agrónomos

Trueba, I. (1985). Proyectos agrarios y desarrollo Rural: Formulación. Madrid: ETSI Agrónomos

Trueba, I. (1995). Formulación y evolución de proyectos empresariales. Madrid: Mundiprensa

Trueba, I. (2002). La seguridad alimentaria mundial. Madrid: Cátedra Alfonso Martín Escudero

Trueba, I. (2011). Concepto de Proyecto: Lecciones de experiencia. In: XV Congreso Internacional de Ingeniería de Proyectos. Huesca

Wolf, M. (1981). Elusive Development. General United Nations Research Institute for Social Development. 